\title{
First experience with the St Jude Medical, Inc, Symmetry Bypass System (Aortic Connector System)
}

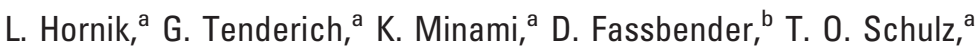 \\ B. Beinert, ${ }^{a}$ and R. Koerfer, ${ }^{a}$ Bad Oeynhausen, Germany
}

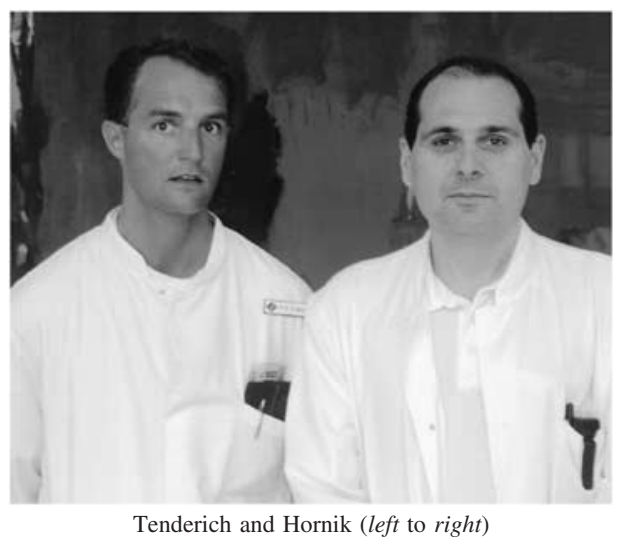

$\mathrm{U}$ sing the saphenous vein graft in coronary artery bypass grafting, the proximal anastomosis is usually performed by running sutures with a partial occlusion clamp. This technique is bearing the risk of immediate or delayed aortic dissection induced by the heel of a partial occlusion clamp, ${ }^{1}$ especially in the case of a diseased aortic wall or poor suturing technique.

If atheroma or calcification of the aortic wall are present, clamping is often impossible, and it remains unclear whether the use of the partial occlusion clamps on the pulsating aorta during off-pump surgery will increase the risk of iatrogenic injuries.

However, the St Jude Medical, Inc, Symmetry Bypass System (Aortic Connector System [ACS]) presents an alternative way of creating the proximal anastomosis of saphenous vein grafts. ${ }^{2}$ It avoids aortic bite clamping and reduces the need for aortic manipulation.

\section{Patients and Methods}

Between April and October 2001, 69 of 70 proximal anastomoses were consecutively performed in 45 patients aged 36 to 89 years (mean, 68.3 years) by using the ACS. Eighteen internal thoracic artery grafts, 70 autologous vein grafts, and 1 radial artery graft were used, for a total number of 108 peripheral anastomoses with an average of 2.4 anastomoses per patient. Five patients were operated on in a conventional manner with the heart-lung machine. Of 31 off-pump patients, 9 patients had to be switched to on-pump therapy, with a conversion rate of $29 \%$.

From the Department of Thoracic and Cardiovascular Surgery a and the Department of Cardiology, ${ }^{\mathrm{b}}$ Heart Center North Rhine-Westfalia, RuhrUniversity of Bochum, Bad Oeynhausen, Germany.

Received for publication April 3, 2002; accepted for publication May 30, 2002.

Address for reprints: Lech Hornik, Heart Center North Rhine-Westphalia, Department of Thoracic and Cardiovascular Surgery, Georgstrasse 11, Bad Oeynhausen North Rhine-Westphalia 32545, Germany.

J Thorac Cardiovasc Surg 2003;125:414-7

Copyright $\odot 2003$ by The American Association for Thoracic Surgery $0022-5223 / 2003 \$ 30.00+0$

doi: $10.1067 / \mathrm{mtc} .2003 .137$
In 4 patients with postoperatively de novo angina pectoris, coronary angiography was indicated, showing severe stenosis of the proximal anastomoses in each case.

In all cases the stenosis appeared several weeks after discharge from the hospital. Three patients underwent a redo procedure using the heart-lung machine and partial occlusion clamp, and one patient was treated by means of dilatation.

PATIENT 1. A 52-year-old woman had a history of dialysis since 1998 and 3-vessel disease. Preoperatively, she was treated with nitrate and heparin because of unstable angina pectoris after 2 fruitless attempts at angioplasty of the right coronary artery (RCA). There was no myocardial infarction. Ejection fraction was $74 \%$. Elective on-pump coronary artery surgery was performed on June 28, 2001, and indication for use of the ACS was a severe calcified ascending aorta. Two single-vein grafts to the left anterior descending coronary artery (LAD) and the posterior descending coronary artery (PDA) were placed. A short time after discharge, the patient had angina pectoris. Cardiac catheterization (September 19, 2001) showed an occlusion of the PDA bypass next to the stenosed proximal ACS anastomosis (Figure 1), as well as a subtotal stenosis of the proximal anastomosis of the ACS-LAD graft (Figure 2). On September 21, the patient had an acute inferior myocardial infarction and a maximum creatine kinase level of 400 U/L. Redo surgery was performed successfully on October 18, 2001, in a conventional manner by using cardiopulmonary bypass and placing 2 single-vein grafts to the RCA and the marginal branch of the circumflex artery, as well as the left internal thoracic artery to the LAD. Histopathologic examination of the proximal parts of the explanted PDA bypass showed severe intimal fibrosis, as well as organized and partially revascularized thrombosis.

PATIENT 2. A 47-year-old woman was given a diagnosis of 2-vessel disease. She had a history of myocardial infarction, but her ejection fraction was normal. The operation was performed on April 24, 2001, placing 2 single-vein grafts to the RCA and diagonal branch $(\mathrm{Dg})$ by means of off-pump coronary surgery with the ACS. The postoperative course was without any complications until September 2001, when the patient again had angina pectoris. Cardiac catheterization showed a severe stenosis of the proximal anastomosis of both vein grafts (Figures 3 and 4), and redo surgery was performed successfully on September 20, 2001, placing 2 single-vein grafts to the PDA, as well as to the Dg, in the conventional method with a heart-lung machine and partial clamping. 


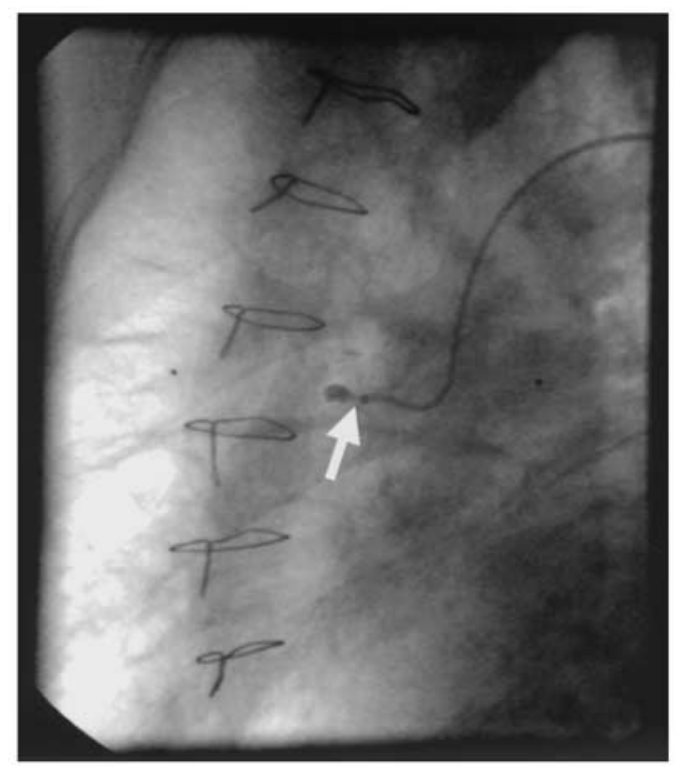

Figure 1. Occlusion of the saphenous vein graft to the posterior descending coronary artery next to the stenosed proximal ACS anastomosis.

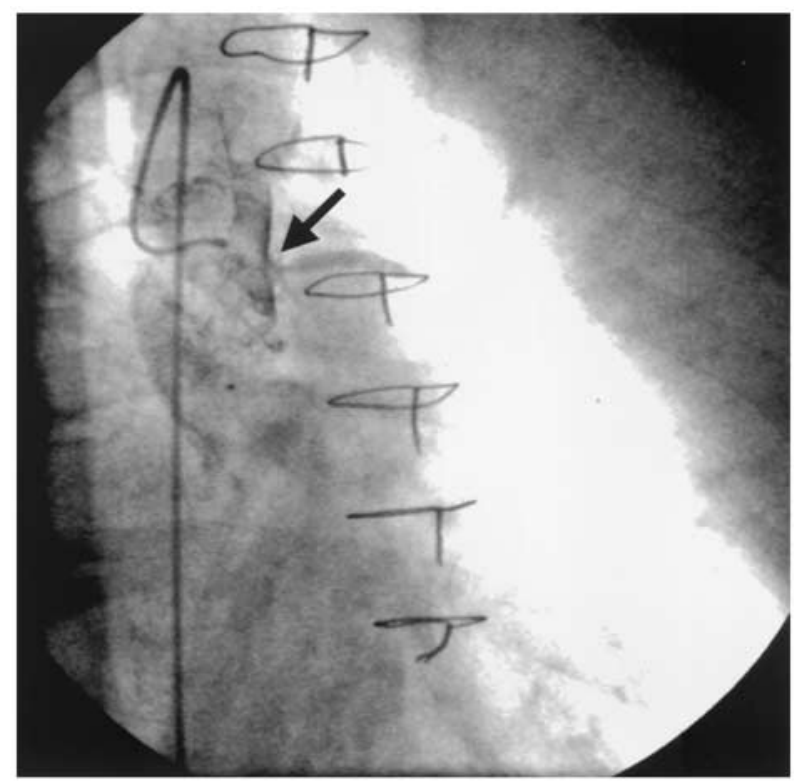

Figure 2. High-grade stenosis of the proximal vein graft anastomosis to the LAD performed with the ACS.

PATIENT 3. A 57-year-old man given a diagnosis of 2-vessel disease underwent off-pump coronary surgery, placing one sequential vein graft to the $\mathrm{Dg}$ and to the proximal as well as the distal part of the LAD (snake graft) on June 9, 2001, with the ACS. The postoperative course was uneventful, and the patient was discharged 6 days after surgical treatment.

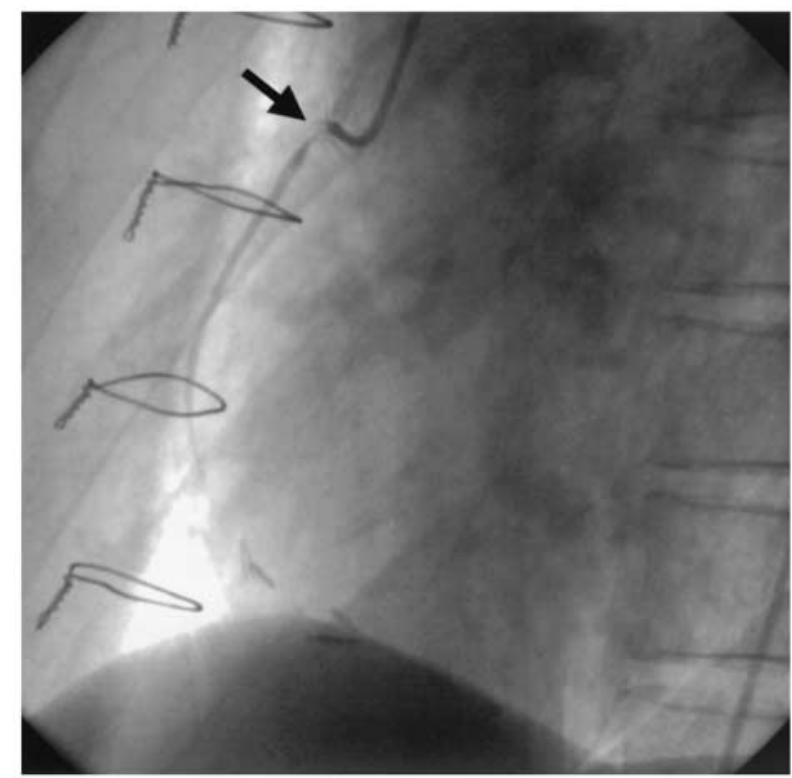

Figure 3. High-grade stenosis of the proximal vein graft anastomosis to the RCA related to the ACS.

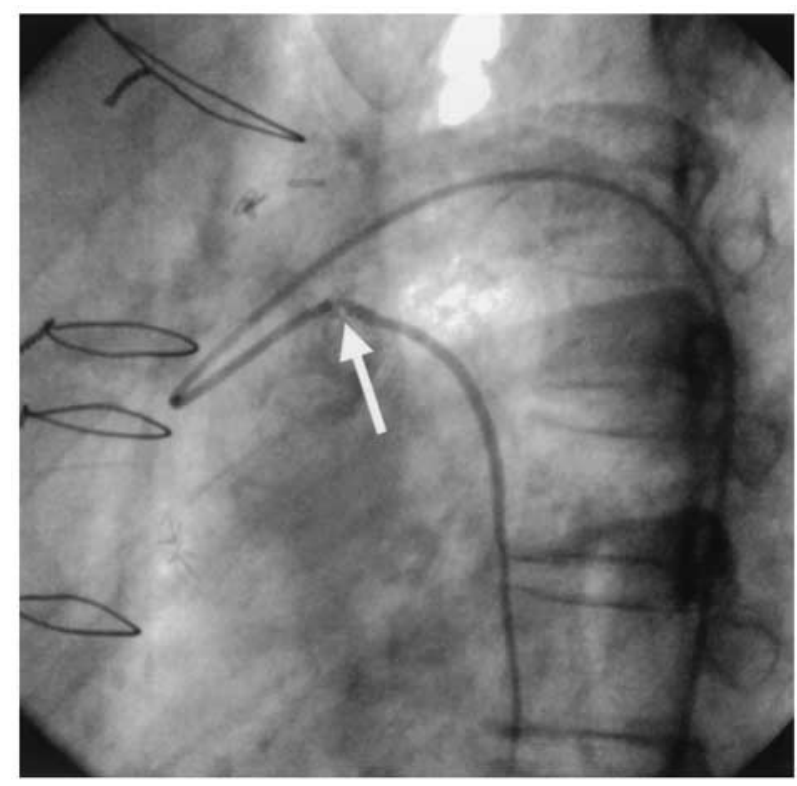

Figure 4. Stenosis of the proximal anastomosis of the saphenous vein graft to the $\mathrm{Dg}$ performed with the ACS.

At the end of 2001, he again had angina pectoris, and cardiac catheterization showed a high-grade stenosis of the proximal (Figure 5) and peripheral anastomosis. Redo surgery was successfully performed on January 7, 2002, by using cardiopulmonary bypass and partial occlusion clamping.

PATIENT 4. A 78-year-old man was given a diagnosis of 2-vessel disease, and he had an ejection fraction of $24 \%$. Preoperatively, 


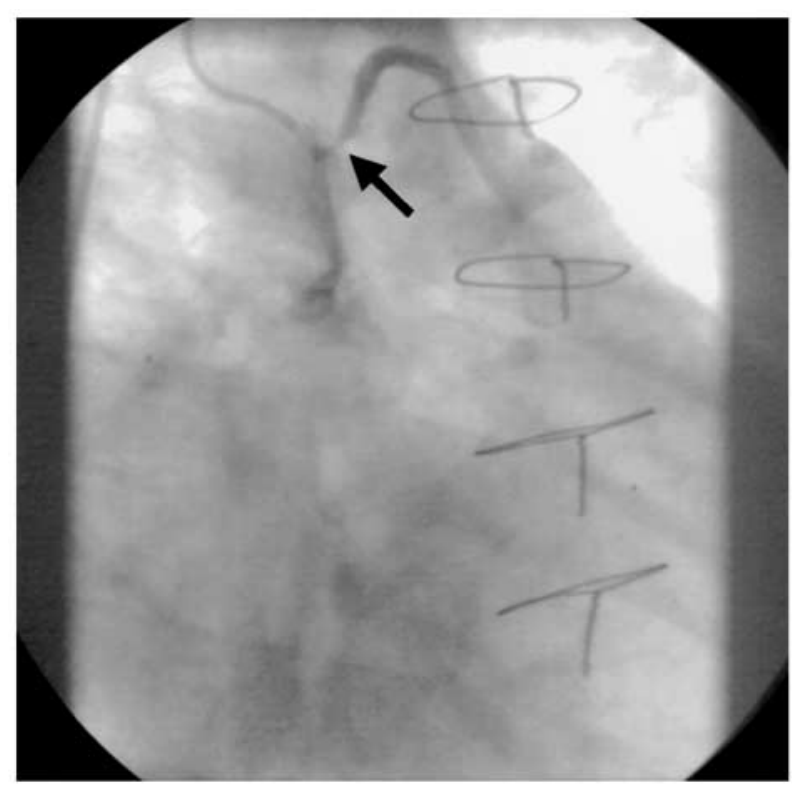

Figure 5. High-grade stenosis of the proximal anastomosis of the sequential LAD-Dg vein graft related to the ACS.

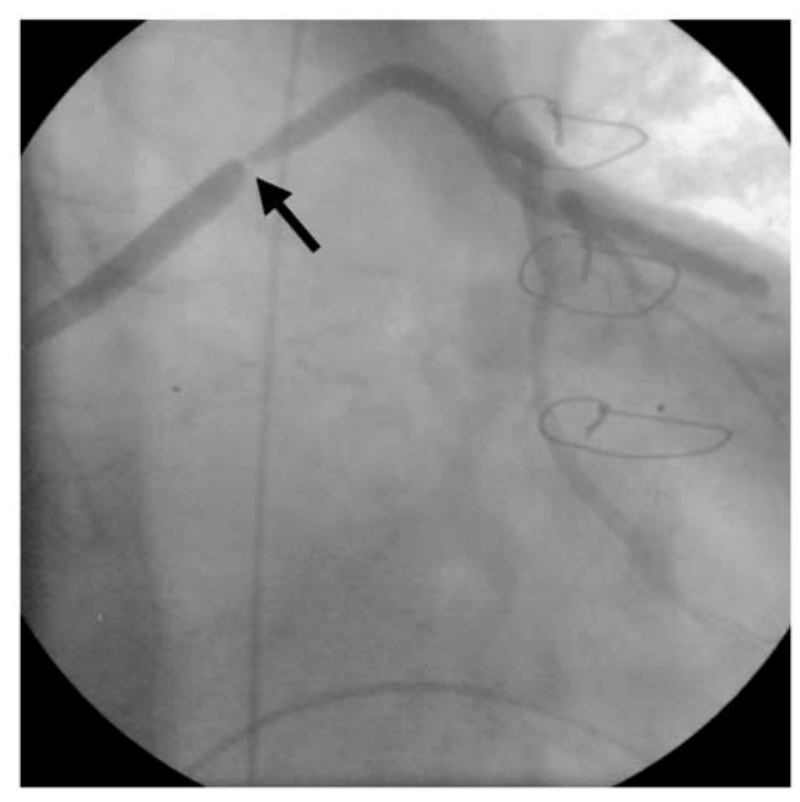

Figure 6. High-grade stenosis of the LAD-Dg graft in the middle part of the bypass proximal to venovenous anastomosis.

the patient reported unstable angina pectoris. Coronary surgery was performed on April 4, 2001, by using conventional cardiopulmonary bypass. A sequential vein graft was placed to the $\mathrm{Dg}$ and LAD by using the ACS for severe calcification of the aorta. A further single graft was placed to the marginal branch of the circumflex artery with a venovenous anastomosis to the LAD-Dg graft. A short time after discharge, he again had angina pectoris.

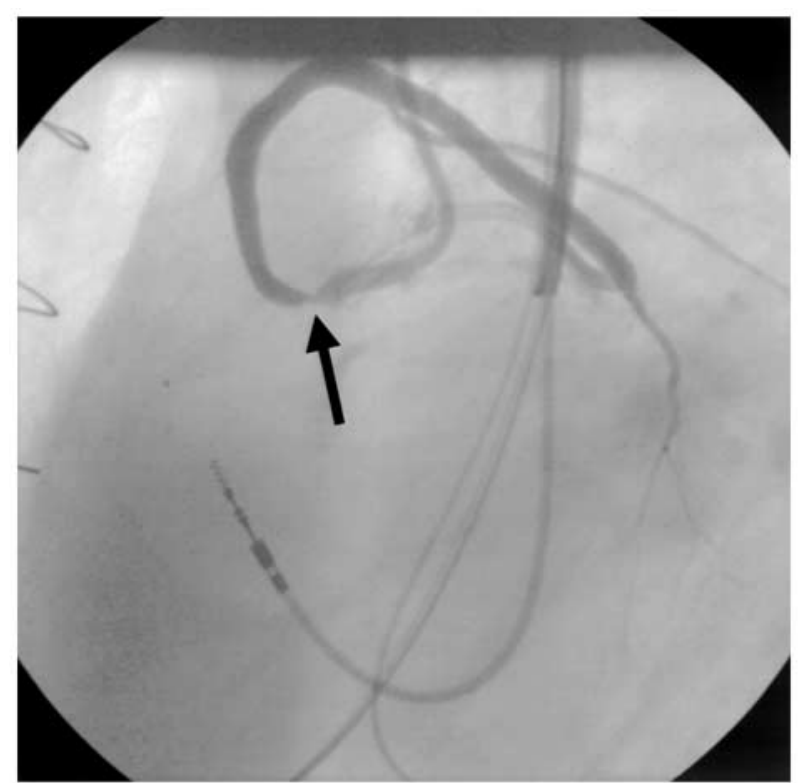

Figure 7. Stenosis of the proximal anastomosis of the LAD-Dg graft related to the ACS.

Cardiac catheterization showed a high-grade stenosis of the LAD-Dg graft proximal to the venovenous anastomosis (Figure 6). Successful dilatation and stenting of the stenosis was done on June 13, 2001. In December 2001, the patient was admitted again because of recurrent unstable angina pectoris. Cardiac catheterization showed a high-grade stenosis of the proximal anastomosis of the LAD-Dg graft related to the ACS (Figure 7), which was dilated and stented successfully on December 7, 2001.

\section{Discussion}

Using a partial occlusion clamp for proximal anastomosis in aortacoronary bypass surgery bears the risk of iatrogenic dissections or embolic complications for various reasons. These complications are more likely in patients with the risk factors of atheroma, calcification, medial necrosis, a thin fragile aorta, or an increased wall tension during off-pump surgery. ${ }^{1}$ St Jude Medical, Inc, offers a novel sutureless connector system for creation of the proximal anastomosis of saphenous vein grafts without the need for clamping of the aorta. ${ }^{2}$ The vein hook is made of nickel titanium (Nitinol), a biocompatible alloy, and published results show that the ACS does not seem to impair endothelial function.*

However, 4 of 45 patients treated with the ACS between April 2001 and October 2001 had a proximal ACS-related stenosis, thrombosis of the saphenous vein graft, or both, with the need for reintervention within 6 months postoperatively. One resected vein graft showed a partially revascularized thrombosis of the proximal graft. Genesis of the stenoses is unknown. We did not observe any kinking of any of the implanted grafts at the aortic take-off point as a probable cause. A possible explanation could be the unavoid-

*Verma S, Ko L, Fedak PWM, Cusimano RJ, Yau T. The St Jude Medical sutureless aortic anastomotic connector does not impair endothelial function in human saphenous veins. Unpublished data. 
able learning curve, resulting in an undersizing or oversizing or the incomplete piercing of the saphenous vein, followed by rolling up of the intima and resulting thereby in graft stenosis or occlusion, mechanical alteration of the intima itself, or bioincompatibility of the device. The significance of the ACS in the genesis of proximal graft stenosis, thrombosis, or both has to be elucidated by means of further investigations.

\section{References}

1. Hagl C, Ergin A, Galla JD, Spielvogel D, Lansman S, Squiteri RP, et al. Delayed chronic type A dissection following CABG. J Card Surg. 2000;15:362-7.

2. Eckstein FS, Bonilla LF, Engelberger L, Stauffer E, Berg TA, Schmidli J, et al. Minimizing aortic manipulation during OPCAB using the symmetry aortic connector system for proximal vein graft anastomoses. Ann Thorac Surg. 2001;72:S995-8.

\section{The last to die is hope: Prolonged mechanical circulatory support with a Novacor left ventricular assist device as a bridge to transplantation}

Dino Casarotto, MD, Tomaso Bottio, MD, Antonio Gambino, MD, Luca Testolin, MD, and Gino Gerosa, MD, Padua, Italy

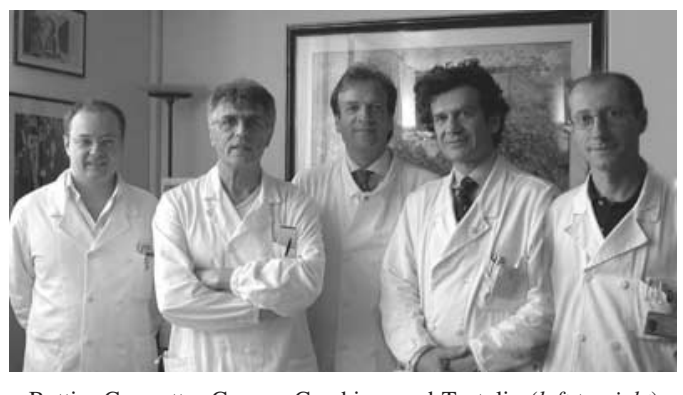

Bottio, Casarotto, Gerosa, Gambino, and Testolin (left to right)

I n September 1996 a 31-year-old man was admitted to Padua Hospital, Padua, Italy, in cardiogenic shock that was not responsive to medical treatment and intra-aortic balloon pump insertion. The patient had Castleman disease, and in 1989 he was treated with cycles of cyclophosphamide, vincristine, mitoxantrone hydrochloride (INN: mitoxantrone), and interferon. In June 1996 at another hospital, he had postchemotherapy dilated cardiomyopathy diagnosed, with akinesia of septum and anterior left ventricle wall (end-diastolic volume $230 \mathrm{~mL}$, end-systolic volume $180 \mathrm{~mL}$, ejection fraction $20 \%$, and left ventricular enddiastolic diameter $67 \mathrm{~mm}$ ). Because of severe left ventricular failure, we started therapy with a BioMedicus left ventricular assist device (Medtronic BioMedicus, Inc, Minneapolis, Minn) and an intra-aortic balloon pump. In October 1996 we converted the centrifugal pump support to a Novacor left ventricular assist system (World Heart Novacor LVAS N100 PCq; World Heart Corporation, Ottawa, Ontario, Canada) because of the impossibility of weaning the patient from the BioMedicus pump and the risks

From the Department of Cardiovascular Surgery, University of Padua Medical School, Padua, Italy.

Received for publication Feb 26, 2002; accepted for publication May 21, 2002.

Address for reprints: Dino Casarotto, MD, Istituto di Chirurgia Cardiovascolare, Via Giustiniani, 1, 35121 Padova, Italy (E-mail: dino. casarotto@unipd.it).

J Thorac Cardiovasc Surg 2003;125:417-8

Copyright (C) 2003 by The American Association for Thoracic Surgery

$0022-5223 / 2003 \$ 30.00+0$

doi: $10.1067 / \mathrm{mtc} .2003 .131$ associated with heart transplantation related to potential relapse of neoplastic disease (chest computed tomographic scan with mediastinal lymph nodes $>2 \mathrm{~cm}$ ).

The Novacor left ventricular assist system, a device used for patients with cardiogenic shock, is considered an interim system to be used before transplantation or to achieve left ventricular recovery for patients who are not candidates for transplantation. ${ }^{1}$ In the long term the main disadvantages of this device are driveline infections, thromboembolism, and system failures affecting the pump and valves. ${ }^{2}$

During the patient's recovery period, he had several episodes of pocket driveline infections and one episode of cerebral embolism, all with complete recovery (no abnormal findings on cerebral computed tomographic scan at follow-up). After the patient had been supported by the Novacor device for 24 months, we attempted to wean him from the system but were unable to do so. In June 2000, the patient showed moderate dyspnea with symptoms of congestive heart failure that necessitated angiotensin-converting enzyme inhibitors and diuretics. A 2-dimensional Doppler echocardiographic evaluation showed moderate incompetence of the Novacor inflow valve. After 50 months of successful mechanical circulatory support (December 5, 2000), there were no signs indicating a relapse of Castleman disease, and we were able to perform orthotopic heart transplantation.

As a result of chemotherapy for Castleman disease, this patient had marked variations in myocardial cells, as seen in the endomyocardial biopsy specimen, with great areas of interstitial fibrosis that caused left ventricular dysfunction. Because of the possibility of myocardial function recovery and the high rate of neoplastic lesions seen in other transplant recipients after immunosuppressive therapy, ${ }^{3}$ rather than perform an orthotopic heart transplant we supported the patient with a mechanical Novacor device. With the 